\title{
Actitud del personal médico de un servicio de pediatría ante la muerte de los pacientes
}

\author{
Luz María Sánchez-Sánchez, Edreí Daniel López-Córdova y Pedro Siller-Gómez \\ Instituto Mexicano del Seguro Social, Centro Médico Nacional del Noreste, Hospital de Especialidades 25. Monterrey, Nuevo León, México
}

\section{Resumen}

Introducción: Los médicos de hospitales de tercer nivel requieren alta competencia emocional, ya que enfrentan las demandas de enfermos terminales y sus familiares. Objetivo: Evaluar la actitud de médicos de un servicio de pediatría ante la muerte de los pacientes. Método: Médicos adscritos y residentes de pediatría contestaron anónimamente el Cuestionario de Actitudes ante la Muerte (CAM). Se utilizaron frecuencias absolutas, porcentajes, medias, desviaciones estándar, $t$ de Student y chi cuadrado. Se consideró significación estadística con $p<0.05$. Resultados: Contestaron el cuestionario 38 médicos, 26 (68.4\%) residentes y 12 (37.6 \%) adscritos; 15 (39.4\%) hombres y 23 (60.6\%) mujeres. Cuatro residentes (15.4 \%) tuvieron actitud positiva versus dos médicos adscritos (16.7\%) ( $p=0.920)$. Se encontró significación estadística en los ítems 1 (69.2 \% de residentes versus $100 \%$ de adscritos, $p=0.03$ ), 16 (23\% de residentes versus $100 \%$ de adscritos, $p=0.001$ ) y 19 (92.3\% de residentes versus $58.3 \%$ de adscritos, $p=0.01$ ), correspondientes a las dimensiones evitación, aceptación y perspectiva profesional. Conclusión: Menos de 20 \% de los médicos de pediatría tiene una actitud positiva ante la muerte. La mayoría no valoró la muerte como un pasaje o tránsito hacia una vida mejor, ni como una salida a los problemas de la vida.

PALABRAS CLAVE: Actitud del médico. Cuidados paliativos. Cuestionario de actitud ante la muerte.

\begin{abstract}
Introduction: Tertiary care hospitals' physicians require high emotional competence, since they are faced with the demands of terminally ill patients and their families. Objective: to assess the attitude of physicians of a pediatrics department towards patient death. Method: Pediatrics staff and resident physicians anonymously answered the Attitudes Towards Death (ATD) questionnaire. Absolute frequencies, percentages, means, standard deviations, Student's t-test and the chi-square test were used. Statistical significance was considered with $p<0.05$. Results: The questionnaire was answered by 38 physicians, 26 (68.4\%) were residents and 12 (37.6\%) staff physicians; 15 (39.4\%) were males and 23 (60.6\%) females. Four residents $(15.4 \%)$ had a positive attitude versus 2 staff physicians (16.7\%) $(p=0.920)$. Statistical significance was found in items 1 (69.2\% of residents versus $100 \%$ of staff physicians, $p=0.03), 16$ (23\% of residents versus $100 \%$ of staff physicians, $p=0.001)$ and 19 (92.3\% of residents versus $58.3 \%$ of staff physicians, $p=0.01)$, corresponding to the avoidance, acceptance and professional perspective dimensions. Conclusion: Less than $20 \%$ of pediatric physicians have a positive attitude towards death. Most didn't value death as a passage or transition to a better life, or as a solution to life's problems.
\end{abstract}

KEY WORDS: Physician's attitude. Palliative care. Attitude towards death questionnaire.

\section{Correspondencia:}

Luz María Sánchez-Sánchez

E-mail: luzsanchez68@ hotmail.com
Fecha de recepción: 22-01-2016

Fecha de aceptación: 30-04-2016

DOI://dx.doi.org/10.24875/GMM.18002358
Gac Med Mex. 2018;154:152-160

Disponible en PubMed www.gacetamedicademexico.com 


\section{Introducción}

En la sociedad actual, se espera que los niños vivan más que sus padres. Sin embargo, los niños también sufren enfermedades crónicas degenerativas que llevan a un estadio terminal que inexorablemente avanza hacia la muerte.

Una enfermedad que limita la vida se define como una situación en la que la muerte prematura es esperable, aunque no necesariamente inminente. Una enfermedad que amenaza la vida es la que tiene una alta probabilidad de muerte prematura, pero en la que también existe la posibilidad de supervivencia hasta la edad adulta. ${ }^{1}$

En los niños se han identificado cuatro grupos de situaciones:

- Grupo 1. Situaciones que amenazan la vida, para las cuales el tratamiento curativo puede ser viable, pero también puede fracasar (por ejemplo, infecciones, cáncer, fallo orgánico del corazón, hígado o riñón).

- Grupo 2. Enfermedades que requieren largos periodos de tratamiento intensivo dirigido a prolongar la vida, pero donde todavía es posible la muerte prematura (por ejemplo, fibrosis quística, $\mathrm{VIH} /$ sida, anomalías cardiovasculares, prematuridad extrema).

- Grupo 3. Enfermedades progresivas sin opciones curativas, en las que el tratamiento es paliativo desde el diagnóstico (por ejemplo, trastornos neuromusculares o neurodegenerativos, trastornos metabólicos progresivos, anomalías cromosómicas, cáncer metastásico avanzado al diagnóstico).

- Grupo 4. Situaciones irreversibles, no progresivas, con grave discapacidad, que conllevan extrema vulnerabilidad de padecer complicaciones de la salud (por ejemplo, parálisis cerebral grave, trastornos genéticos, malformaciones congénitas, prematuridad, lesiones cerebrales o de la médula espinal). ${ }^{2,3}$

La muerte es un verdadero proceso biológico, psicosocial y cultural, no es un fenómeno instantáneo. En los tiempos modernos existe tendencia a la ocultación, a la evasión en el tema de la muerte, mediante censura social rígida, que, incluso, involucra el engaño al moribundo, convirtiéndolo en un ente aislado, lleno de desesperación y con atención insuficiente.

La falta de tiempo, la carencia de formación curricular en técnicas de comunicación, la susceptibilidad propia hacia la representación de la muerte y la inseguridad son factores que proceden del personal de salud y que afectan y hacen más vulnerable al moribundo. El tema de la muerte sigue siendo un tabú, con sus características específicas. ${ }^{4}$

Aun cuando la enfermedad y la muerte constituyen reales posibilidades de la existencia humana, con frecuencia se elude hablar del tema. Las costumbres sociales contemporáneas facilitan esta actitud evasiva. La idea de dejar de existir es rechazada y la muerte se convierte en un acto no propio, temido. Incluso, la actitud que una persona tiene ante la muerte puede tener influencia en su conducta y relacionarse con los demás problemas de esa persona. La muerte se ha convertido en una expresión de miedo, de fracaso en la búsqueda de la felicidad y del éxito. Paradójicamente, la sociedad, que sigue siendo mortal, rechaza la muerte. De hecho, aunque esencial a la existencia humana, la muerte ha pasado a ser un absurdo, padecido en la ignorancia y en la pasividad, en una falta de solidaridad y abandono con respecto a los moribundos, los difuntos y los supervivientes. ${ }^{5}$

La muerte familiar de antaño se ha ido convirtiendo en una muerte ocultada. Ya no se muere en casa sino en el hospital, a solas. La vida obligadamente feliz pretende alejar toda idea de la muerte y las apariencias de "siempre estar vivo" predominan. Se esconde el tema, se le habla de la muerte a los niños mediante eufemismos; el luto se hace cada vez más en la intimidad y se comparte poco el dolor.

Los profesionales en hospitales tienden a concebir la muerte como un fracaso en vez de admitirla como un fenómeno natural necesario. Cada día es más deseable morir sin darse cuenta, pero también conviene morir sin que los demás se den cuenta. Aunque hay diferencias en varios países, e incluso, dentro de un mismo país entre diversas regiones, la mayoría de las personas que enferman crónicamente van a morir al hospital. Ya casi no se muere en el domicilio, rodeado por los seres queridos. El hombre moderno ha ido perdiendo su derecho a ser el protagonista del acto de morir, que se convierte en un proceso cada vez más "técnico", en algo que hay que olvidar rápidamente; se afecta así no sólo la calidad de muerte, sino la calidad de vida de los últimos momentos. No es casual que la Organización Mundial de la Salud (OMS) promueva la idea de que el enfermo debe morir en casa, rodeado de objetos queridos, en una atmósfera tranquila, al lado de sus familiares y allegados, respetando sus derechos y voluntades, después de haber recibido 
durante cierto tiempo los beneficios de los programas de cuidados paliativos..$^{6-9}$

En ocasiones, los especialistas subestiman el entendimiento del niño acerca de su enfermedad, de la muerte y de otros asuntos relacionados con la espiritualidad. Lo niños pueden entender el concepto de la muerte como un estado de transición como a los 3 años de edad; pueden empezar a tener el sentido de la posibilidad de la mortalidad personal a los $8 \circ 9$ años. La espiritualidad y la religiosidad o la piedad pueden ir separadas. Incluso, sin un entorno religioso, todos los niños poseen una vida espiritual..$^{10,11}$

Myra Bluebond-Langner describió por primera vez el "mutuo fingimiento" que frecuentemente no deja a los niños y sus padres comunicarse acerca de la muerte y del hecho de morir. ${ }^{12}$ En Suecia, Kreicberg et al. publicaron recientemente un estudio retrospectivo sobre padres que habían perdido a un hijo por cáncer. De los 147 que informaron haber hablado con su hijo acerca de la muerte, ninguno lo lamentaba, comparado con $27 \%$ de los 258 padres que no habló acerca de ello. Entre los padres que presintieron que su hijo conocía su inminente muerte, la probabilidad de arrepentimiento por no haber hablado de ello fue más alta. Los padres que no hablaron sobre la muerte tenían un nivel de ansiedad más alto durante el seguimiento..$^{13}$

La pérdida de un hijo es uno de los mayores factores de estrés en la vida. Los padres que pierden a un hijo tienen riesgo de sufrir reacciones complejas de duelo, así como de una posible muerte por causas naturales o no naturales. Las reacciones defensivas de la familia de los niños y adolescentes ante una enfermedad crónica y terminal son la culpabilidad, la sobreprotección ansiosa, el rechazo, la negación de la enfermedad y la dependencia y la negación o devaluación de los médicos. Las madres pueden desarrollar depresión mayor, ya que la muerte de un hijo es difícil de aceptar. ${ }^{14}$

Para los profesionales de la salud, en particular para los médicos, el tema de la muerte involucra un análisis complejo, porque engloba toda su actitud hacia el paciente; les resulta difícil sostener una actitud serena y madura frente a ella. Cuando sucede, enseguida tratan de sentirse bien, actitud que traduce una forma de ocultamiento, un mecanismo de defensa ante el dolor. Se supone que el profesional de la salud, y el médico en particular, es el más íntegramente educado por, para y contra la muerte, vista como una realidad biológica, un fenómeno humano y un valor cultural. Sin embargo, no es siempre así, lo que con frecuencia se refleja en la poca atención a los enfermos incurables. ${ }^{15-18}$

En primer lugar, existe un déficit de formación, ya que no se enseña lo que hay que hacer con enfermos incurables y esto no se compensa con el buen deseo de ayudar, pero da lugar a una situación defensiva del profesional, a que responda a la ansiedad con mecanismos de defensa inadecuados: cólera, actitudes negativas, etcétera. En segundo lugar, la muerte constituye para los profesionales de la salud, especialmente para los médicos, una sensación de fracaso profesional; se les enseña a salvar vidas, y aunque saben que la muerte es inevitable, tienden a sentirse culpables de no ser capaces de curar a sus enfermos. En tercer lugar, la confrontación con la muerte del paciente les genera angustia ante su propia muerte, pues también son seres humanos y en escenarios como éste afloran prejuicios y creencias, ansiedades y la historia personal de cada uno. ${ }^{19}$

Todo profesional de la salud debería tener interés en analizar y comprender los diferentes componentes de su malestar: una toma de conciencia de las razones ocultas que le empujan a huir ante tales situaciones le permitiría rectificar su actitud y estar más cómodo en semejantes circunstancias. De cualquier manera, la mayoría de las veces el enfermo terminal y su familia se encuentran bajo un gran impacto y generan un ambiente con gran carga emocional, en la que hay que saber estar; el profesional de la salud debe prepararse y formarse para ello, aprender que la muerte es algo natural. Cuando es capaz de aceptarla así, se dedicará a cuidar y consolar a su enfermo hasta el final y sin sensación de fracaso. ${ }^{20-22} \mathrm{La}$ actitud del médico es vital para el manejo adecuado de los enfermos, y en gran medida condiciona la actitud de estos ante la proximidad de su muerte.

Está claro que debe gestarse el desarrollo de actitudes adecuadas ante la muerte 0 el cambio significativo de actitudes hacia ella, un movimiento de comprensión y respeto hacia las circunstancias de la muerte, en las que equipos multidisciplinarios aborden a pacientes y familiares.

\section{Método}

De forma anónima, a médicos adscritos y residentes de pediatría del Servicio de Pediatría, Hospital de Especialidades 25, Centro Médico Nacional del Noreste, Instituto Mexicano del Seguro Social, en Monterrey, Nuevo León, se les aplicó el Cuestionario de 
Actitudes ante la Muerte (CAM), que obtuvo un alpha de Cronbach de 0.713 al ser previamente validado.

El CAM está compuesto por 33 reactivos, con 2 alternativas de respuesta (acuerdo o desacuerdo), que puede dar un total de 33 a 165 puntos; 141 puntos o más corresponde a una actitud positiva hacia la muerte y puntuaciones inferiores a actitud negativa. Para su interpretación está dividido en seis dominios que corresponden a diferentes actitudes ante la muerte: de evitación, de aceptación, de temor, basadas en las creencias de que la muerte es un pasaje o tránsito, fundamentadas en la concepción de la muerte como una salida o solución y otra que involucra la perspectiva profesional (anexo 1). ${ }^{20,22}$ Cada dominio fue evaluado por separado entre los grupos de profesionales de la salud del servicio de pediatría.

En el consentimiento informado se garantizó el anonimato de los participantes, dado que la actitud sobre un tema implica una postura personal y es deseable que los participantes se sientan en completa libertad de expresarla sin ser identificados.

Para el análisis se utilizaron frecuencias absolutas y porcentajes, así como medias o medianas con desviaciones estándar o rangos. Para comparar las puntuaciones obtenidas entre los médicos adscritos y residentes se utilizó la $t$ de Student $o$ la $U$ de Mann-Whitney. Para variables cualitativas se utilizó chi cuadrado o prueba exacta de Fisher. Se consideró significación estadística con $\mathrm{p}<0.05$.

El presente estudio cumple con las consideraciones formuladas en la Declaración de Helsinki y sus modificaciones de Tokio en 1975, Venecia en 1983 y Hong Kong en 1989; además, se apega a las consideraciones formuladas en la investigación para la salud de la Ley General de Salud de los Estados Unidos Mexicanos y al instructivo para la operación de la comisión de investigación científica y de los comités locales de investigación del Instituto Mexicano del Seguro Social.

\section{Resultados}

Treinta y ocho médicos aceptaron contestar el CAM, que consta de 33 ítems. Del total de los encuestados, $26(68.4 \%)$ eran médicos residentes y 12 (37.6 \%) médicos adscritos; 15 (39.4\%) eran hombres y $23(60.6 \%)$ mujeres. La edad de los residentes fue de $28.1 \pm 1.6$ años y de $44.9 \pm 10.3$ años en los médicos adscritos $(p=0.001)$. La antigüedad laboral fue de $2.8 \pm 1.6$ años en los residentes y de $15.2 \pm 8.5$ años en los adscritos $(p=0.001)$.
La puntuación total del cuestionario fue de $120 \pm 16.4$ en los residentes y de $133 \pm 14.4$ en los médicos adscritos $(p=0.05)$. En relación con las dimensiones del cuestionario, la evitación ante la muerte fue de $21 \pm 4.7$ puntos para los médicos residentes en comparación con $25 \pm 2.0$ en los adscritos ( $p=0.086$ ); en aceptación se encontró $21 \pm 5.6$ puntos en los residentes y $23 \pm 4.5$ en los adscritos $(p=0.090)$; en cuanto al temor a la muerte se obtuvieron $21 \pm 6.1$ puntos en los residentes y $21 \pm 6.9$ en los adscritos ( $p=0.908)$. En relación con los que creen que la muerte es un pasaje, las puntuaciones obtenidas por los residentes fue de $17 \pm 7.5$ y $21 \pm 5.7$ en los médicos adscritos $(p=0.304)$. Los que ven la muerte como una salida, la puntuación fue de $21 \pm$ 3.03 en los residentes y en los adscritos de $21 \pm 4$ $(p=0.980)$; al valorar la perspectiva profesional de los médicos residentes se obtuvo una puntuación de $28 \pm 4.07$ y para los médicos adscritos de $28 \pm 4.55$ $(p=0.116)$. La puntuación total en el CAM por arriba de 141 se considera actitud positiva ante la muerte; cuatro residentes (15.4\%) tuvieron actitud positiva comparado con dos adscritos $(16.7 \%)(p=0.920)$ (Tabla 1).

En la Tabla 2, los ítems fueron agrupados por dimensión y analizados de manera individual en médicos adscritos y residentes. Se encontró diferencia

Tabla 1. Características demográficas y puntuaciones obtenidas en el Cuestionario de Actitud ante la Muerte por 38 médicos*

\begin{tabular}{lccc}
\hline & $\begin{array}{c}\text { Residentes } \\
(\mathbf{n}=\mathbf{2 6})\end{array}$ & $\begin{array}{c}\text { Adscritos } \\
(\mathbf{n}=\mathbf{1 2})\end{array}$ & $\mathbf{p}$ \\
\hline Sexo & & & \\
$\quad$ Masculino & $8(30.7 \%)$ & $7(58.3 \%)$ & 0.920 \\
$\quad$ Femenino & $18(69.3 \%)$ & $5(41.6 \%)$ & \\
& $28.1 \pm 1.6$ & $44.9 \pm 10.3$ & 0.001 \\
Edad (años) & $2.8 \pm 0.8$ & $15.2 \pm 8.5$ & 0.001 \\
Antigüedad (años) & $120 \pm 16.4$ & $133 \pm 14.4$ & 0.050 \\
Actitud hacia la & & & \\
muerte (puntuación) & & & \\
& & & \\
Dimensión (puntuación) & & & \\
$\quad$ Evitación & $21 \pm 4.7$ & $25 \pm 2.0$ & 0.086 \\
$\quad$ Aceptación & $21 \pm 5.6$ & $23 \pm 4.5$ & 0.090 \\
$\quad$ Temor & $21 \pm 6.1$ & $21 \pm 6.9$ & 0.908 \\
Pasaje & $17 \pm 7.5$ & $21 \pm 5.7$ & 0.304 \\
$\quad$ Salida & $21 \pm 3.0$ & $21 \pm 4.0$ & 0.980 \\
Perspectiva profesional & $28 \pm 4.0$ & $28 \pm 4.5$ & 0.116 \\
& & & \\
Actitud hacia la muerte & & & \\
$\quad$ Positiva & & & \\
Negativa & $4(15.4 \%)$ & $2(16.7 \%)$ & 0.920 \\
\hline
\end{tabular}

*Servicio de Pediatría, Hospital de Especialidades 25, Monterrey, Nuevo León. Valores expresados en media \pm desviación estándar o frecuencias absolutas (porcentaje). 
Tabla 2. Resultados obtenidos en cada ítem del Cuestionario de Actitud ante la Muerte por $\mathbf{3 8}$ médicos*

\begin{tabular}{|c|c|c|c|c|c|c|c|}
\hline \multirow[t]{2}{*}{ Ítem } & \multirow[t]{2}{*}{ Dimensión } & & \multicolumn{2}{|c|}{ Residentes $(n=26)$} & \multicolumn{2}{|c|}{ Adscritos $(n=12)$} & \multirow[t]{2}{*}{$\mathrm{p}$} \\
\hline & & & De acuerdo & Desacuerdo & De acuerdo & Desacuerdo & \\
\hline 2 & A & $\begin{array}{l}\text { La aceptación de la muerte me ayuda a tener } \\
\text { más responsabilidad ante la vida. }\end{array}$ & $21(80.8 \%)$ & $5(19.2 \%)$ & $11(91.7 \%)$ & $1(8.3 \%)$ & 0.39 \\
\hline 7 & A & $\begin{array}{l}\text { Mi vida tiene más significado porque yo acepto } \\
\text { el hecho de mi propia muerte. }\end{array}$ & $14(53.8 \%)$ & $12(46.2 \%)$ & $8(66.7 \%)$ & $4(33.3 \%)$ & 0.46 \\
\hline 16 & A & $\begin{array}{l}\text { He pensado en mi muerte como un hecho } \\
\text { inevitable. }\end{array}$ & $6(23.1 \%)$ & $20(76.9 \%)$ & $12(100 \%)$ & - & 0.00 \\
\hline 23 & A & $\begin{array}{l}\text { Reconocer la muerte como un hecho inevitable } \\
\text { me ayuda a mi crecimiento personal. }\end{array}$ & $18(69.2 \%)$ & $8(30.7 \%)$ & $9(75 \%)$ & $3(25 \%)$ & 0.70 \\
\hline 27 & A & Me siento más libre al aceptar mi muerte. & $16(61.5 \%)$ & $10(38.4 \%)$ & $11(91.6 \%)$ & $1(8.3 \%)$ & 0.06 \\
\hline 1 & $E$ & Pensar en la muerte es perder el tiempo. & $8(30.8 \%)$ & $18(69.2 \%)$ & - & $12(100 \%)$ & 0.03 \\
\hline 8 & $E$ & $\begin{array}{l}\text { Considero morboso pensar deliberadamente en } \\
\text { mi muerte inevitable. }\end{array}$ & $23(88.5 \%)$ & $3(11.5 \%)$ & $9(75 \%)$ & $3(25 \%)$ & 0.20 \\
\hline 17 & $E$ & Yo realmente prefiero no pensar en la muerte. & $9(34.6 \%)$ & $17(65.3 \%)$ & $2(16.6 \%)$ & $10(83.3 \%)$ & 0.20 \\
\hline 22 & $E$ & $\begin{array}{l}\text { No he pensado hasta ahora en la muerte como } \\
\text { una posibilidad real. }\end{array}$ & $6(23.1 \%)$ & $20(76.9 \%)$ & - & $12(100 \%)$ & 0.07 \\
\hline 32 & $E$ & $\begin{array}{l}\text { Las personas solo deben pensar en la muerte } \\
\text { cuando son viejos. }\end{array}$ & $3(11.5 \%)$ & $23(88.4 \%)$ & 0 & $12(100 \%)$ & 0.22 \\
\hline 4 & $\mathrm{P}$ & $\begin{array}{l}\text { Yo pienso que después de la vida hay un lugar } \\
\text { mejor. }\end{array}$ & $18(69.2 \%)$ & $8(30.8 \%)$ & $10(83.3 \%)$ & $2(16.7 \%)$ & 0.36 \\
\hline 10 & $P$ & Pienso que viviré después de mi muerte. & $16(61.5 \%)$ & $10(38.4 \%)$ & $8(66.6 \%)$ & $4(33.3 \%)$ & 0.76 \\
\hline 18 & $\mathrm{P}$ & Veo la muerte como un paso a la eternidad. & $16(61.5 \%)$ & $10(38.4 \%)$ & $11(91.6 \%)$ & $1(8.3 \%)$ & 0.06 \\
\hline 25 & $P$ & $\begin{array}{l}\text { Yo espero con placer la vida después de la } \\
\text { muerte. }\end{array}$ & $11(42.3 \%)$ & $15(57.6 \%)$ & $6(50 \%)$ & $6(50 \%)$ & 0.60 \\
\hline 29 & $\mathrm{P}$ & Después de la muerte encontraré felicidad. & $11(42.3 \%)$ & $15(57.6 \%)$ & $7(58.3 \%)$ & $5(41.6 \%)$ & 0.36 \\
\hline 6 & PP & $\begin{array}{l}\text { Siento que la muerte de mi paciente es un } \\
\text { fracaso profesional. }\end{array}$ & $3(11.5 \%)$ & $23(88.5 \%)$ & - & $12(100 \%)$ & 0.22 \\
\hline 11 & PP & $\begin{array}{l}\text { No quisiera asistir al episodio terminal de un } \\
\text { paciente de mi especialidad. }\end{array}$ & $1(3.8 \%)$ & $25(95.1 \%)$ & $2(16.6 \%)$ & $10(83.3 \%)$ & 0.17 \\
\hline 12 & PP & $\begin{array}{l}\text { No puedo evitar pensar en mi familia ante un } \\
\text { paciente que va a morir. }\end{array}$ & $11(42.3 \%)$ & $15(57.6 \%)$ & $8(66.6 \%)$ & $4(33.3 \%)$ & 0.16 \\
\hline 19 & $\mathrm{PP}$ & $\begin{array}{l}\text { Cuando atiendo a un paciente terminal, me } \\
\text { pongo a pensar en mi propia muerte. }\end{array}$ & $2(7.6 \%)$ & $24(92.3 \%)$ & $5(41.6 \%)$ & $7(58.3 \%)$ & 0.01 \\
\hline 20 & PP & $\begin{array}{l}\text { Me conmueve enfrentar a la familia de un } \\
\text { paciente moribundo. }\end{array}$ & $21(80.7 \%)$ & $5(19.2 \%)$ & $9(75 \%)$ & $3(25 \%)$ & 0.60 \\
\hline 26 & PP & $\begin{array}{l}\text { El más alto sentido de mi trabajo es salvar la } \\
\text { vida del paciente. }\end{array}$ & $17(65.3 \%)$ & $9(34.6 \%)$ & $8(66.6 \%)$ & 4 (33.3 \%) & 0.90 \\
\hline 30 & PP & $\begin{array}{l}\text { El contacto frecuente con la muerte me ha hecho } \\
\text { verla como algo natural. }\end{array}$ & $21(80.7 \%)$ & $5(19.2 \%)$ & $11(91.6 \%)$ & $1(8.3 \%)$ & 0.39 \\
\hline 33 & PP & $\begin{array}{l}\text { Me siento más cómodo si la petición de asistir } \\
\text { a un paciente moribundo proviene de sus } \\
\text { familiares. }\end{array}$ & $17(65.3 \%)$ & $9(34.6 \%)$ & 8 (66.6 \%) & $4(33.3 \%)$ & 0.90 \\
\hline
\end{tabular}


Tabla 2. Resultados obtenidos en cada ítem del Cuestionario de Actitud ante la Muerte por 38 médicos* (Continuación)

\begin{tabular}{|c|c|c|c|c|c|c|c|}
\hline \multirow[t]{2}{*}{ Ítem } & \multirow[t]{2}{*}{ Dimensión } & & \multicolumn{2}{|c|}{ Residentes ( $n=26$ ) } & \multicolumn{2}{|c|}{ Adscritos $(n=12)$} & \multirow[t]{2}{*}{ p } \\
\hline & & & De acuerdo & Desacuerdo & De acuerdo & Desacuerdo & \\
\hline 5 & S & $\begin{array}{l}\text { La muerte puede ser una salida a la carga de } \\
\text { la vida. }\end{array}$ & $3(11.5 \%)$ & $23(88.5 \%)$ & $3(25 \%)$ & $9(75 \%)$ & 0.29 \\
\hline 14 & S & $\begin{array}{l}\text { Pienso que ya no hay nada que ver en este } \\
\text { mundo. }\end{array}$ & - & $26(100 \%)$ & - & $12(100 \%)$ & 0.99 \\
\hline 15 & S & Prefiero morir a vivir sin calidad & $15(57.6 \%)$ & $11(42.3 \%)$ & $4(33.3 \%)$ & $8(66.6 \%)$ & 0.16 \\
\hline 21 & S & He pensado que no vale la pena vivir. & $1(3.8 \%)$ & $25(96.1 \%)$ & - & $12(100 \%)$ & 0.49 \\
\hline 31 & S & $\begin{array}{l}\text { Hay momentos que la muerte puede ser un } \\
\text { alivio. }\end{array}$ & $20(76.9 \%)$ & $6(23.1 \%)$ & $10(83.3 \%)$ & $2(16.6 \%)$ & 0.60 \\
\hline 3 & $\mathrm{~T}$ & $\begin{array}{l}\text { La posibilidad de mi propia muerte me despierta } \\
\text { ansiedad. }\end{array}$ & $6(23.1 \%)$ & $20(76.9 \%)$ & $5(41.7 \%)$ & $7(58.3 \%)$ & 0.24 \\
\hline 9 & $\mathrm{~T}$ & $\begin{array}{l}\text { Me siento perturbado cuando pienso en lo corta } \\
\text { que es la vida. }\end{array}$ & $5(19.2 \%)$ & $21(80.8 \%)$ & - & $12(100 \%)$ & 0.10 \\
\hline 13 & $\mathrm{~T}$ & Yo temo morir joven. & $3(11.5 \%)$ & $23(88.4 \%)$ & $4(33.3 \%)$ & $8(66.6 \%)$ & 0.10 \\
\hline 24 & $\mathrm{~T}$ & Encuentro difícil encarar la muerte. & $8(30.7$ \%) & $18(69.2 \%)$ & $3(25 \%)$ & $9(75 \%)$ & 0.70 \\
\hline 28 & $\mathrm{~T}$ & $\begin{array}{l}\text { Pienso con temor ante la posibilidad de contraer } \\
\text { una enfermedad que me lleve a la muerte. }\end{array}$ & $12(46.1 \%)$ & $14(53.8 \%)$ & $6(50 \%)$ & $6(50 \%)$ & 0.80 \\
\hline
\end{tabular}

*Servicio de Pediatría, Hospital de Especialidades 25, Monterrey, Nuevo León.

A, aceptación; E, evitación; P, pasaje; P, perspectiva profesional; S, salida; T, temor.

significativa en el ítem 1 (pensar que la muerte es perder el tiempo), que corresponde a la dimensión evitación: 18 residentes $(69.2 \%)$ contestaron estar en desacuerdo en comparación con los $12(100 \%)$ médicos adscritos $(p=0.03)$. En el ítem 16 (he pensado en la muerte como algo inevitable), que corresponde a la aceptación de la muerte, seis residentes (23\%) estuvieron de acuerdo comparados con 12 adscritos $(100 \%)(p=0.00)$. En el ítem 19 (cuando atiendo a un paciente terminal, me pongo a pensar en mi propia muerte), que pertenece a la dimensión de perspectiva profesional, $24(92.3 \%)$ residentes indicaron desacuerdo en comparación con siete $(58.3 \%)$ de los médicos adscritos $(p=0.01)$. No hubo diferencia estadísticamente significativa con los otros ítems del cuestionario.

Se notó ambigüedad de respuesta con porcentajes similares de acuerdo y desacuerdo en el ítem 7 (mi vida tiene más significado porque yo acepto el hecho de mi propia muerte), que corresponde a la aceptación. En la dimensión de pasaje, el cual se basa en las creencias de que la muerte es un pasaje o tránsito hacia otra vida existió más ambigüedad de respuesta, ya que los porcentajes de acuerdo y desacuerdo fueron similares en 4 de sus 5 ítems. En la dimensión de perspectiva profesional hubo ambigüedad de respuesta en ítem 11 (no puedo evitar pensar en mi familia ante un paciente que va a morir). También se observó ambigüedad en el ítem 14 (prefiero morir sin calidad), que corresponde a la dimensión de salida, y en el ítem 28 (pienso con temor ante la posibilidad de contraer una enfermedad que me lleve a la muerte), que corresponde a la dimensión de temor.

\section{Discusión}

La muerte no es un fenómeno instantáneo, es un proceso biológico, psicosocial y cultural. A pesar de que la enfermedad y la muerte constituyen posibilidades reales de la existencia humana, con frecuencia se elude hablar del tema. La conciencia de muerte es una característica fundamentalmente humana; las actitudes, creencias, sentimientos y comportamientos del hombre ante la muerte siguen siendo fenómenos psicosociales, culturalmente aprendidos y reforzados a lo largo de la vida.

Para los profesionales de la salud, y en particular los médicos, el tema de la muerte involucra un análisis complejo, porque engloba toda su actitud hacia el paciente; es difícil sostener una actitud serena y madura frente a ella. Cuando sucede, enseguida tratan de sentirse bien, lo que constituye una forma de ocultamiento, un mecanismo de defensa ante el dolor que produce. Se supone que son los profesionales 
más íntegramente educados por, para y contra la muerte, vista como una realidad biológica, un fenómeno humano y un valor cultural. ${ }^{4}$

El Hospital de Especialidades 25, del Instituto Mexicano del Seguro Social, en Monterrey, Nuevo León, proporciona atención médica a pacientes procedentes de la zona noreste del país, brindando atención a niños, la mayoría con diagnósticos de enfermedades crónicas, incapacitantes o catastróficas para la vida, por lo que el conocimiento del personal salud acerca de tener una actitud positiva hacia la muerte es esencial para brindar una atención de calidad. ${ }^{1}$

Se han identificado numerosas barreras para tener una actitud positiva ante la muerte: insuficientes recursos financieros para el cuidado pediátrico especializado, acceso limitado a cuidados especiales, problemas de comunicación, falsas esperanzas de curación, problemas éticos, religiosos y legales, criterios de inclusión inapropiados, evaluación y manejo inadecuados de la sintomatología y, la principal, falta de entrenamiento del personal de salud. ${ }^{6}$

Diversos estudios han demostrado que los conocimientos y las habilidades reflejadas en la actitud acerca de la muerte pueden ser mejorados. Por lo tanto, el primer paso es identificar la actitud del personal que labora en el Servicio de Pediatría del Hospital de Especialidades 25, para la cual se aplicó el Cuestionario de Actitudes ante la Muerte (CAM), que pone de manifiesto la postura de cada persona)..$^{20,22}$

En las etapas en la formación del médico, la actitud ante la muerte se atribuye a un fracaso en la actividad profesional y no a un fenómeno natural necesario. La medicina paliativa no figura en las prioridades de salud en México; en las instituciones existe gran desconocimiento sobre las necesidades físicas psicológicas, sociales y espirituales del paciente en estado terminal y sobre las de su familia; se reconoce que los profesionales de la salud carecen de los conocimientos necesarios sobre cuidados paliativos y como resultado tienen una actitud negativa ante la muerte de los pacientes. ${ }^{7-9}$

En este estudio se incluyeron 38 médicos, incluyendo médicos residentes que se encontraban haciendo la especialidad de pediatría y médicos adscritos del servicio. Los médicos adscritos obtuvieron puntuaciones más altas que los médicos residentes, lo que denota una mejor actitud ante la muerte, sin embargo, estos promedios estaban por debajo de los 141 puntos que se consideran actitud positiva. Al analizar las puntuaciones por dominios, no hubo diferencias estadísticas entre las obtenidas por residentes y adscritos.
Menos de $20 \%$ de los médicos residentes y adscritos tuvo actitud positiva ante la muerte, lo cual no resulta sorprendente dado que hay un déficit de formación, ya que el médico tiende a ver la muerte como un enemigo, y las escuelas de medicina refuerzan involuntariamente esta ilusión. ${ }^{3}$ Los médicos consideran que su trabajo es luchar contra la muerte, aun sabiendo que es inevitable. La muerte constituye para los profesionales de la salud, especialmente para los médicos, una sensación de fracaso profesional; tiende a sentirse culpable de no ser capaz de curar a su enfermo y la situación terminal es entendida como signo de impotencia, muchas veces inconscientemente. El profesional debe aprender que la muerte es algo natural. Cuando es capaz de aceptarla así, se dedicará a cuidar y consolar a su enfermo hasta el final y sin sensación de fracaso. ${ }^{9-11}$

En un estudio realizado por Hernández Cabrera, donde se aplicó el CAM a 50 médicos de familia, se hizo evidente que las actitudes en la población estudiada manifestaban cierta ambigüedad (ambivalencia direccional) junto a algunas actitudes positivas de aceptación (especialmente a nivel cognitivo), predominio de actitudes de evitación, matizadas fundamentalmente por el contenido afectivo (temor). La mayoría de los médicos no valoró la muerte como un pasaje o tránsito hacia una vida mejor, ni como una salida a los problemas de la vida, aunque gran parte reconoció que en determinados momentos puede ser un alivio al sufrimiento.

Los resultados en este estudio realizado a 38 médicos de pediatría indican diferencia significativa entre residentes y adscritos en un ítem de aceptación que explora la muerte como algo inevitable y en otro ítem de evitación que habla sobre pensar en la muerte, en el que los residentes se muestran más reacios a pensar en ella, tal vez debido a que estos son más jóvenes y asocian la muerte con la vejez. También hubo diferencia significativa en el ítem 19, que habla sobre pensar en la propia muerte cuando se atiende a un paciente terminal; en este ítem son los médicos adscritos los que tienden a pensar más en su propia muerte que los residentes. Sin embargo, fue notoria la ambigüedad o ambivalencia direccional en la dimensión de pasaje, tal como se encontró en el estudio de Hernández Cabrera. ${ }^{22}$

En un estudio realizado por Leticia Ascencio Huertas, en el Instituto Nacional el Cancerología de México, se aplicó el CAM a 31 personas que laboraban en un equipo multidisciplinario de cuidados paliativos oncológicos, entre los que se encontraban 
médicos, psicólogos, enfermeras; se identificaron porcentajes por ítem muy similares a los encontrados en este estudio, incluso también es evidente la ambigüedad en las respuestas de la dimensión de pasaje, ya que este tiene que ver con las creencias de cada persona en relación con la muerte como un pasaje a otra vida. ${ }^{20}$

Ayudar a los enfermos a morir en paz se reconoce como una tarea ante todo humana y digna de elogio, pero permanece como un objetivo médico de menor categoría que vencer a la muerte y solo se admite cuando fallan por completo los medios para evitarla. La muerte no es un fracaso del conocimiento y del ejercicio médico, no puede retrasarse indefinidamente, es el fin natural e inevitable de la existencia que nos afectará a todos. Los grandes avances y descubrimientos médicos ayudarán a preservar la salud y alargar la vida del hombre, pero no podrán evitar que las personas sigan muriendo. Los profesionales de la salud deberían sentirse tan inclinados a investigar y tratar el sufrimiento que frecuentemente acompaña al proceso de morir o a la pérdida de un ser querido, como para investigar los factores y mecanismos que pueden ayudar a prolongar la vida. En un proceso de reestructuración del concepto y fines de la medicina son dos las metas y ambas tienen la misma categoría y la misma importancia: evitar la muerte cuando se pueda y lograr que los pacientes vivan bien hasta final, que fallezcan en paz, cuando ya lo primero no es posible. $^{22}$

La educación es un componente esencial en el proceso de desarrollar una cultura positiva ante la muerte de los pacientes pediátricos, por lo tanto, el personal de la salud necesita ser entrenado para tener una actitud positiva, la cual se verá reflejada en la alta calidad de atención para los niños y sus familias. ${ }^{1,2}$

\section{Conclusión}

1. Menos de $20 \%$ de los médicos de pediatría tiene una actitud positiva ante la muerte.
2. La mayoría de los médicos no valoró la muerte como un pasaje o tránsito hacia una vida mejor, ni como una salida a los problemas de la vida, aunque gran parte de ellos reconoció que en determinados momentos puede ser un alivio al sufrimiento.

\section{Bibliografía}

1. American Academy of Pediatrics. Committee on Bioethics and Committee on Hospital Care. Palliative care for children. Pediatrics. 2000;106(2 Pt 1):351-357.

2. Garduño-Espinoza A. Cuidados paliativos en niños. Atención a pacientes con enfermedad terminal. Acta Pediatr Mex. 2004;25:1-3.

3. Garduño-Espinoza A. Cuidados paliativos en el niño con enfermedad terminal. Dol Clin Ter. 2003:9(1);5-10.

4. Málishev-Krasnova M. El sentido de la muerte. Ciencia Ergo Sum. 2003;10(1):51-58.

5. Aries P. Historia de la muerte en occidente. Barcelona: El Acantilado; 2000.

6. Gómez-Sancho M. Morir con dignidad. Madrid: Arán Ediciones; 2005.

7. Gómez-Sancho R. Medicina paliativa en la cultura latina. Madrid: Arán Ediciones; 1999.

8. Gala-León FJ, Lupiani-Jiménez M, Raja-Hernández R, Guillén-Gestoso C, González-Infante JM, Villaverde-Gutiérrez MC, et al. Actitudes psicológicas ante la muerte y el duelo. Una revisión conceptual. Cuad Med Forense. 2002;30:39-50.

9. Fonnegra I. La muerte, el morir y el deudo desde diferentes perspectivas. Bogotá: Fundación Omega; 1992.

10. Hilden JM, Watterson J, Chrastek J. Tell the children. J Clin Oncol. 2000;18(17):3193-3195.

11. Hynson JL, Sawyer SM. Pediatric palliative care: distinctive needs and emerging issues. J Pediatr Child Health. 2001;37(4):323-325.

12. Blueband-Lagner M. The private lives of dying children. USA: Princeton University Press; 1978.

13. Kreicbergs U, Valdimarsdóttir U, Onelöv E, Björk O, Steineck G Henter JI. Care-related distress: a nationwide study of parents who lost their child to cancer. J Clin Oncol. 2005;23(36):9162-9171.

14. Lannen PK, Wolfe J, Prigerson HG, Onelov E, Kreicbergs UC. Unresolved grief in a national sample of bereaved parents: impaired mental and physical health 4 to 9 years later. J Clin Oncol. 2008;26(36):5870-5876.

15. Davies B, Sehring SA, Partridge C, Cooper BA, Hughes A, Philp JC, et al. Barriers to palliative care for children: perceptions of pediatric health care providers. Pediatrics. 2008;121(2):282.

16. Burns JP, Mitchell C, Griffith JL, Truog RD. End-of-life care in the pediatric intensive care unit: attitudes and practices of pediatric critical care physicians and nurses. Crit Care Med. 2001;29(3):658-664.

17. Khaneja S, Milrod B. Educational needs among pediatricians regarding caring for terminally ill children. Arch Pediatric Adolesc Med. 1998:152(9):909-914.

18. Pichardo-García LM, Diner K. La experiencia de la muerte y los cuidados paliativos. Una visión desde enfermería. An Med (Mex). 2010; 55(3):161-166.

19. Brockopp DY, King DB, Hamilton JE. The dying patient: a comparative study of nurse caregiver characteristics. Death Stud. 1991;15(3):245-258.

20. Ascencio-Huertas L, Allende-Pérez SR, Verastegui-Avilés E. Creencias, actitudes y ansiedad ante la muerte en un equipo multidisciplinario de cuidados paliativos oncológicos. Psicooncología. 2014;11(1):101-115.

21. Lara K, Ruiz N, Balcázar P. Ansiedad ante la muerte en adultos de población general y personal que trabaja en el sector salud. Revista Científica Electrónica de Psicología ICSa-UAEH. 2009:4:83-106.

22. Hernández-Cabrera G, González-García VM, Fernández-Machín L, Infante-Pereira $O$. Actitud ante la muerte en los médicos de la familia. Rev Cubana Med Gen Integr. 2002;18(1):22-32. 
Anexo 1. Cuestionario de Actitudes ante la Muerte

\begin{tabular}{|c|c|}
\hline \multicolumn{2}{|c|}{ E-evitación, A-aceptación, T-temor, P-pasaje, S-salida, PP-perspectiva profesional } \\
\hline $1 \mathrm{E}$. & Pensar en la muerte es perder el tiempo. \\
\hline $2 A$. & La aceptación de la muerte me ayuda a tener más responsabilidad ante la vida. \\
\hline ЗТ. & La posibilidad de mi propia muerte me despierta ansiedad. \\
\hline $4 \mathrm{P}$. & Yo pienso que después de la vida hay un lugar mejor. \\
\hline $5 \mathrm{~S}$. & La muerte puede ser una salida a la carga de la vida. \\
\hline 6PP. & Siento que la muerte de mi paciente es un fracaso profesional. \\
\hline $7 A$. & Mi vida tiene más significado porque yo acepto el hecho de mi propia muerte. \\
\hline $8 \mathrm{E}$. & Considero morboso pensar deliberadamente en mi muerte inevitable. \\
\hline 9T. & Me siento perturbado cuando pienso en lo corta que es la vida. \\
\hline 10P. & Pienso que viviré después de mi muerte. \\
\hline $11 \mathrm{PP}$. & No quisiera asistir al episodio terminal de un paciente de mi especialidad. \\
\hline 12PP. & No puedo evitar pensar en mi familia ante un paciente que va a morir. \\
\hline 13T. & Yo temo morir joven. \\
\hline $14 \mathrm{~S}$. & Pienso que ya no hay nada que ver en este mundo. \\
\hline $15 S$. & Prefiero morir a vivir sin calidad. \\
\hline $16 \mathrm{~A}$. & He pensado en mi muerte como un hecho inevitable. \\
\hline $17 \mathrm{E}$. & Yo realmente prefiero no pensar en la muerte. \\
\hline $18 \mathrm{P}$. & Veo la muerte como un paso a la eternidad. \\
\hline 19 PP. & Cuando atiendo a un paciente terminal, me pongo a pensar en mi propia muerte. \\
\hline 20PP. & Me conmueve enfrentar a la familia de un paciente moribundo. \\
\hline $21 S$. & He pensado que no vale la pena vivir. \\
\hline 22E. & No he pensado hasta ahora en la muerte como una posibilidad real. \\
\hline $23 \mathrm{~A}$. & Reconocer la muerte como un hecho inevitable me ayuda a mi crecimiento personal. \\
\hline $24 \mathrm{~T}$. & Encuentro difícil encarar la muerte. \\
\hline 25P. & Yo espero con placer la vida después de la muerte. \\
\hline 26PP. & El más alto sentido de mi trabajo es salvar la vida del paciente. \\
\hline $27 \mathrm{~A}$. & Me siento más libre al aceptar mi muerte. \\
\hline 28T. & Pienso con temor ante la posibilidad de contraer una enfermedad que me lleve a la muerte. \\
\hline 29P. & Después de la muerte encontraré felicidad. \\
\hline 30PP. & El contacto frecuente con la muerte me ha hecho verla como algo natural. \\
\hline $31 \mathrm{~S}$. & Hay momentos que la muerte puede ser un alivio. \\
\hline 32E. & Las personas solo deben pensar en la muerte cuando son viejos. \\
\hline 33PP. & $\begin{array}{l}\text { Me siento más cómodo si la petición de asistir a un paciente moribundo proviene de sus } \\
\text { familiares. }\end{array}$ \\
\hline
\end{tabular}

